Quand j'ai voulu vérifier mes tubes de Gerber, je n'ai pu trouver d'indications certaines sur la base de cette graduation; la maison qui les fabrique n'a pu ou n'a pas voulu m'éclairer à cet égard; mes vérifications ont reposé sur des données personnelles; nul doute que cette absolue confiance à donner au fabricant, constitue un défaut de premier ordre.

Telles sont les raisons qui me paraissent amplement suffisantes, dans leur ensemble, pour justifier l'abandon complet du procédé de Gerber, tout au moins en France où nous avons la réputation d'aimer le simple et le concis.

\title{
PHÉNOMĖNES \\ DE DIFFUSION DES CONSTITUANTS DU LAIT DANS UN GEL DE GÉLOSE
}

\author{
( $2^{e}$ communigation) \\ Par le Dr Alb. J.-J. VANDEVELDE \\ Professeur à l'Institut agronomique et à l'Université de Gand.
}

Dans ma première communication (1), j'ai constaté qu'un gel de gélose n'est pas en état de s'opposer au passage de colloïdes comme les lactoprotéines, et que sans modifier l'état colloïdal du sol une partie des substances' solubles diffuse du sol vers le gel; les constituants du lait restent ainsi dans le même état dispersé que dans le lait lui-même. Après un certain temps, le gel de gélose contient les constituants solubles en équilibre de concentration avec le sol.

Dans cette communication, j'ai annoncé également que je projetais de mettre les sols de concentration diminuée au contact de nouveaux gels de gélose, de manière à diluer encore plus les constituants solubles, et à pouvoir établir, dans ce cas, l'état des protéines. J'ai fait cette recherche et j'ai aussi étudié l'influence exercée par du lactose et par du chlorure de sodium introduits dans le gel à diverses concentrations.

I. - J'ai essayé de reproduire sur du lait sans formol les phénomènes de diffusion afin de voir si le formol additionné exerçait une influence. J'ai fait usage, comme auparavant, de fioles plates de Soyka de $10 \mathrm{~cm}$. de diamètre ; dans chaque fiole j'ai introduit $50 \mathrm{cc}$. de gel de gélose à $2 \%$. Les fioles étaient réunies entre elles de manière à pouvoir faire passer, au moment voulu, aseptiquement le lait d'une fiole dans l'autre ; dans ce but, la première fiole porte un bouchon muni de deux tubes dont l'un est bouché d'ouate et destiné à exercer une pression dans la fiole, et l'autre parvient jusqu'au fond

(1) Le Lait, 1924, 4, p. 638-640. 
de la fiole pour conduire jusqu'au-dessous de la fermeture de la deuxième fiole de la même série; la deuxième fiole porte trois tubes, l'un en communication avec la première fiole, le deuxième va du fond de la fiole jusqu'au-dessous du bouchon de la troisième fiole de la série, le troisième est bouché d'ouate et maintient la pression à l'intérieur de la fiole en équilibre avec la pression extérieure, et ainsi de suite pour la troisième et la quatrième fiole d'une même série. Je comptais pouvoir, de cette manière, faire passer le lait d'une fiole dans l'autre à l'abri des mierobes de l'air atmosphérique.

La première fiole de la série contenait $50 \mathrm{cc}$. de lait centrifugé et pas de gel de gélose, les fioles suivantes contenaient $50 \mathrm{cc}$. de gel de gélose à $2 \%$; toute la série était stérilisée à $100^{\circ} \mathrm{C}$. par trois chauffages consécutifs.

En même temps, et pour eomparaison, jai opéré comme dans les essais décrits dans ma première communieation, en me servant de lait centrifugé formolé $(0,5 \mathrm{cc}$. de formol à $30 \%$ pour $100 \mathrm{ce}$. de lait); j'ai mis en œuvre, par fiole, $50 \mathrm{cc}$. de gel de gélose et $50 \mathrm{ec}$. de lait.

Les fioles étaient conservées horizontales, de manière à maintenir le lait régulièrement au contact avec la couche de gélose. Malheureusement je n'ai pu dépasser, pour le lait non formolé, une période d'un mois ; au bout de deux mois, quand j'ai voulu opérer le deuxième transvasement du lait, j'ai constaté que le lait, dans les diverses séries, était infecté. Mais, par contre, j'ai eu l'occasion de remarquer qu'il n'y avait guère de différence entre le lait non formolé et le lait formolé, ce qui me permet d'admettre que les résultats obtenus avec le lait formolé sont aussi ceux du lait non formolé. En faisant passer, après un intervalle d'un mois chaque fois, le lait dans des fioles contenant du gel de gélose nouveau pour pousser plus avant la diffusion, je suis arrivé aux résultats suivants :

\begin{tabular}{|c|c|c|c|c|c|c|c|}
\hline Dans 100 cc. gr. & & Extrait & Lactose & Azote & $\begin{array}{l}\text { Proteines } \\
(\mathrm{N} \times 6,5)\end{array}$ & Cendres & $\begin{array}{r}\mathrm{PO} 3 \\
-\end{array}$ \\
\hline $\begin{array}{l}\text { Lait initial } \\
\text { Après } 1 \text { mo }\end{array}$ & & 8,58 & 4,56 & 0,47 & 3,05 & 0,71 & 0,1236 \\
\hline Sans formol. & 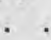 & 5,96 & 2,09 & 0,42 & 2,73 & 0,47 & 0,0854 \\
\hline Avec formol. & & 5,94 & 2, & 0,40 & 2,60 & 0,46 & 0,0716 \\
\hline Après 2 mois. & & 4,39 & 1,02 & 0,36 & 2,34 & 0,36 & 0,0696 \\
\hline Après 3 mois . & & 2,98 & 0,40 & 0,31 & 2,01 & 0,27 & 0,0404 \\
\hline Après 4 mois. & . & 2,22 & 0,10 & 0,25 & 1,62 & 0,24 & 0,0340 \\
\hline
\end{tabular}

Après quatre passages successifs avec intervalles d'un mois, j'ai done obtenu un liquide dans lequel les protéines, ramenées à la moitié environ de la concentration initiale, sont encore en parfait état colloïdal; le lactose est presque complètement éliminé, les matières minérales sont ramenées à environ au tiers de la concentration initiale. Le lactose est réellement en solution véritable et nullement sous la dépendance des autres constituants; les matières 
minérales, et notamment les phosphates, sont partiellement en relation avec les protéines, ce qui concorde avec les hypothèses actuellement en cours.

II. Gels de gélose contenant du lactose. - J'ai étudié la diffusion du lait centrifugé dans les gels de gélose lactosés; les gels contenaient outre $2 \%$ de gélose, respectivement $2,25 \%, 4,50 \%$ et $6,75 \%$ de lactose. Dans chaque fiole de Soyka, j'ai introduit 50 cc. de gel, et puis sur le gel stérilisé et maintenu horizontal, j'ai versé $50 \mathrm{cc}$. de lait centrifugé formolé.

Les dosages effectués après un, deux et trois mois ont conduit aux mêmes résultats : l'extrait diminue, jusqu'à un minimum atteint déjà après un mois quand le gel de gélose ne contient plus de lactose ; si le gel de gélose contient du lactose, la teneur en lactose diminue quand la teneur du gel est de $2,25 \%$, et elle augmente quand cette teneur est de $6,75 \%$. La vitesse de la diminution de la teneur en lactose du sol est retardée par la présence de $2,25 \%$ de lactose dans le gel. Pour la concentration à $4,5 \%$ de lactose, l'équilibre de concentration se maintient entre le gel et le sol. Les. protéines semblent peu influencées par la présence et la concentration du lactose dans le gel; il en est de même pour les cendres et les phosphates.

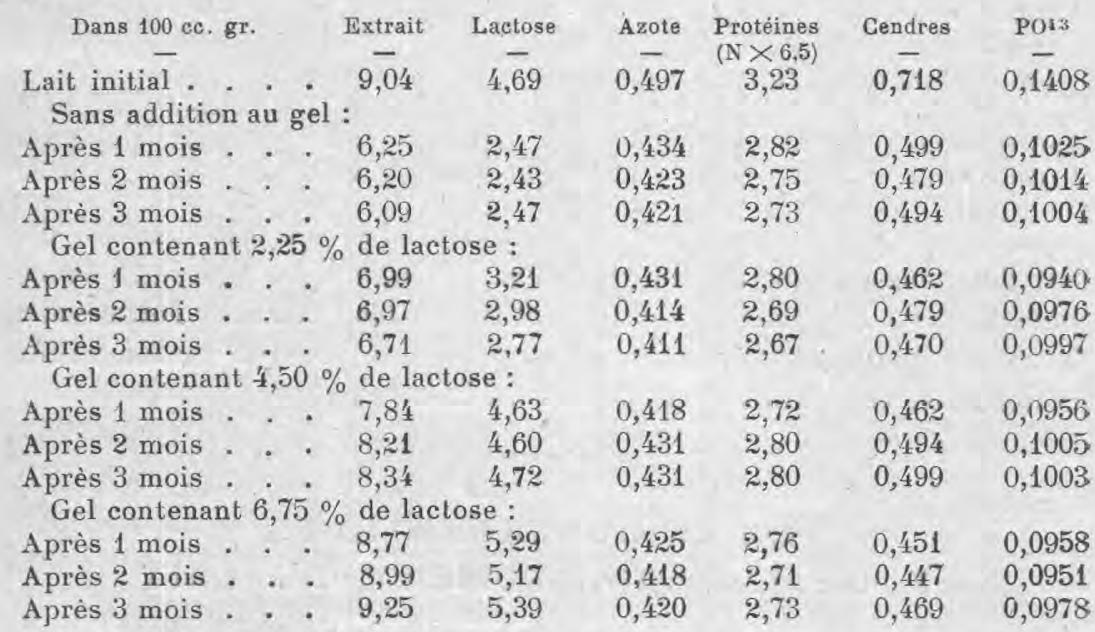

III. Gels de gélose contenant du chlorure de sodium. - Le point de congélation du lait normal se trouve aux environs de - $0056 \mathrm{C}$.; une solution de chlorure de sodium pur de concentration $1 \%$ a un point de congélation de - 0589 C. C'est pourquoi j'ai employé des gels de gélose à $0,5 \%, 1 \%$ et $1,5 \%$ de chlorure de sodium pour réaliser des concentrations osmotiques correspondant à $0,5,1$ et 1,5 de la concentration osmotique du lait. Les dosages des constituants 
du lait dans le sol furent faits après un, deux et trois mois : la teneur èn extrait diminue, le plus pour une teneur initiale de $0,5 \%$, le moins pour $1,5 \%$, vraisemblablement en raison de la diffusion du sel hors du gel vers le sol. La teneur en lactose subit une diminution régulière, comme s'il n'y avait pas de sel dans le gel. Comme dans les essais avec le lactose, les protéines ne sont pas influencées; les cendres diminuent pour une concentration de $0,5 \%$ et augmentent pour les concentrations $1 \%$ et $1,5 \%$, ce qu'il fallait prévoir; la teneur en phosphates subit une diminution régulière en dehors de l'influence de la concentration du gel en chlorure de sodium.

\begin{tabular}{|c|c|c|c|c|c|c|c|}
\hline Dans $100 \mathrm{cc}$. & & Extrait & Lactose & Azote & $\begin{array}{l}\text { Protéines } \\
(\mathrm{N} \times 6,5)\end{array}$ & Cendres & $\mathrm{PO}^{4} 3$ \\
\hline $\begin{array}{l}\text { Lait initial . } \\
\text { Sans addition }\end{array}$ & in au & gel : & 4,69 & 0,497 & 3,23 & 0,718 & 0,1408 \\
\hline Après 1 mois . & . . & 6,25 & 2,47 & 0,434 & 2,82 & 0,499 & 0,1025 \\
\hline Après 2 mois . & . & 6,20 & 2,43 & 0,423 & 2,75 & 0,479 & 0,1014 \\
\hline $\begin{array}{l}\text { Après } 3 \text { mois } \\
\text { Gel contenan }\end{array}$ & at 0,5 & $\begin{array}{c}6,09 \\
\% \mathrm{NaCl} \text { : }\end{array}$ & 2,47 & 0,421 & 2,73 & 0,494 & 0,1004 \\
\hline Après 1 mois . & .. & . 6,43 & 2,47 & 0,452 & 2,94 & 0,642 & 0,1046 \\
\hline Après 2 mois. & . . & 6,41 & 2,22 & 0,429 & 2,79 & 0,639 & 0,0893 \\
\hline $\begin{array}{l}\text { Après } 3 \text { mois } \\
\text { Gel contenan }\end{array}$ & it $i$ & $\begin{array}{r}6,39 \\
\mathrm{NaCl}:\end{array}$ & 1,97 & 0,414 & 2,69 & 0,652 & 0,0890 \\
\hline Après 1 mois . & . . & 6,45 & 2,19 & 0.442 & 2,87 & 0,906 & 0,1025 \\
\hline Après 2 mois. & . . & 6,40 & 1,99 & 0,423 & 2,75 & 0,906 & 0,0995 \\
\hline $\begin{array}{l}\text { Après } 3 \text { mois } \\
\text { Gel contenan }\end{array}$ & & $\begin{array}{c}6,49 \\
\% \mathrm{NaCl}:\end{array}$ & 2,03 & 0,416 & 2,70 & 0,89 ᄂ & 0,0888 \\
\hline Après 1 mois . & . . & - $\quad 6,96$ & 2,09 & 0,427 & 2,77 & 1,051 & 0,1023 \\
\hline Après 2 mois . & . : & 7,05 & 1,96 & 0,428 & 2,78 & 1,020 & 0,0994 \\
\hline Après 3 mois . & . . & 6,92 & 2,00 & 0,409 & 2,66 & 1,208 & 0,0870 \\
\hline
\end{tabular}

Il résulte de ces différentes recherches que les constituants du lait diffusent dans le gel de gélose, et que même les protéines et les phosphates s'intéressent à cette diffusion; les protéines conservent leur état collö̈dal, même quand elles restent presque seules, après la diffusion des autres constituants du lait.

\title{
DE LA CONSERVATION ET DE L'ASSAINISSEMENT DU LAIT
}

\author{
Par le $D^{r}$ Henri STASSANO
}

- suite -

Dans les premiers centres de ramassage du lait qui s'établirent à la campagne pour le ravitaillement des villes voisines, on ne tarda pas à sentir le besoin de chauffer le lait avant de l'expédier au loin, pour en retarder l'acidification naturelle pendant le voyage et surtout pendant le séjour du lait chez le débitant. 